\title{
Implicit priming reveals decomposed processing in fraction comparison
}

\author{
Jessica A. Nejman ${ }^{1}$ \& Thomas J. Faulkenberry ${ }^{1}$ \\ 1 Tarleton State University
}

\begin{abstract}
Fractions present a unique challenge in early mathematics instruction, as they require focusing not on the individual symbols that make up the fraction, but rather a mental combination of the two into a single numerical magnitude. Previous studies have given conflicting accounts of how adults form these complex mental representations. Whereas some studies indicate that mental representations of fractions are holistic and are based upon the fraction's numerical magnitude, others have indicated support for decomposed processing, where separate representations of the numerator and denominator are formed. In the present study, we tested this decomposed processing account using an implicit priming paradigm. In a series of experimental trials, the comparison of two fraction magnitudes ("which is larger?") primed a subsequent comparison trial with whole numbers. Using Bayesian analyses, we found that when people compared two fractions with common denominators, they were faster in the subsequent whole number comparison. However, when two fractions with common numerators were compared, the subsequent whole number comparison was slower. This indicates that representations of the fraction components were activated in the fraction comparison, and these residual activations primed the subsequent whole number comparison. These data give further support to the notion of decomposed processing in fraction comparison.
\end{abstract}

As a core part of American curriculum, mathematics is an area where students struggle. This is especially true for fraction and decimal operations, where difficulties persist from early high school (Hoffer, Venkataraman, Hedberg, \& Shagle, 2007) and carry into adulthood

This research was supported in part by funding from the Tarleton State University Office of Student Research and Creative Activities. The results presented in this manuscript were previously presented at a meeting of the Southwestern Psychological Association.

Correspondence concerning this article should be addressed to Thomas J. Faulkenberry, Department of Psychological Sciences, Box T-0820, Tarleton State University, Stephenville, TX 76401. E-mail: faulkenberry@tarleton.edu 
(Kutner et al., 2007). Kutner et al. (2007) also demonstrated that almost a quarter of adults scored below basic level on simple judgements involving decimals and percentages. This rate of innumeracy has created questions about the cognitive underpinnings of fractions, as such knowledge would surely assist with developing more effective teaching of fractions during school. Since fractions contain multiple symbols which map to an overall numerical magnitude, it is not clear how children move from focusing on the individual symbols (i.e., the numerator and denominator) to mentally forming the ratio of the two numbers. So how do adults do this successfully? Unfortunately, there are relatively few studies which focus on how adults think about fractions, and these studies tend to present conflicting results.

Presently, the literature reveals two main types of fraction processing: holistic and decomposed (Faulkenberry \& Pierce, 2011; Huber, Moeller, \& Nuerk, 2014; Meert, Grégoire, \& Noël, 2009; Obersteiner, Dooren, Hoof, \& Verschaffel, 2013). Some studies suggest that adults use holistic processing in tasks involving fractions (Ganor-Stern, Karasik-Rivkin, \& Tzelgov, 2011; Meert et al., 2009; Meert, Grégoire, \& Noël, 2010; Obersteiner et al., 2013; Obersteiner \& Tumpek, 2015; Schneider \& Siegler, 2010). Holistic processing involves forming mental representations of fractions that involve the fraction's numerical magnitude as a ratio (i.e., $1 / 4=0.25)$ rather than separately activating representations of the components of the fraction (i.e., the numerator 1 and denominator 4). Meanwhile, other studies indicate that fraction tasks are completed using decomposed processing (Bonato, Fabbri, Umiltà, \& Zorzi, 2007; Faulkenberry, Montgomery, \& Tennes, 2015; Faulkenberry \& Pierce, 2011; Gabriel et al., 2013; Huber et al., 2014; Zhang, Fang, Gabriel, \& Szücs, 2014; Zhang et al., 2012), where separate mental representations are formed for the fraction's numerator and denominator. In the majority of studies designed to investigate the nature of fraction representations, the primary task used is numerical comparison, where participants are presented with pairs of fractions and asked to quickly indicate which is the larger fraction. Researchers then investigate how the resulting response times differ as a function of the distance between the compared numbers. In one of the earliest studies on this topic, Bonato et al. (2007) found that response times were better predicted by the numerical distance between component magnitudes than the numerical distance between the fraction magnitudes. From this, they concluded that their participants were using a decomposed, component-based strategy (i.e., comparing numerator to numerator or denominator to denominator), which allowed them to compare the fractions without having to access the numerical magnitude.

In a contrasting study, Schneider \& Siegler (2010) demonstrated holistic processing of fractions. In their experiments, Schneider and Siegler varied the types of fraction stimuli that were presented and made it more difficult for participants to engage in component-based strategies. For example, instead of presenting fraction pairs that differed only in their denominators (e.g., $1 / 3$ versus $1 / 5$ ), Schneider and Siegler used fraction pairs where both components differed (e.g., $3 / 5$ versus $2 / 9$ ). The result was that numerical distance between the fractions (not the components themselves) was the best predictor of response times. Further, Faulkenberry \& Pierce (2011) found that when participants were asked for strategy reports after every trial, there were a wide range of reported strategies, and the type of processing that was employed depended strongly on the nature of these strategies. For example, those who reported using cross-multiplication (a common strategy taught in US schools) exhibited signatures of component-based processing, whereas those who reported 
using mental visualization strategies (e.g., pizza or number line) exhibited holistic processing. As such, it is not clear whether fraction processing type is dependent on the fractions themselves, or rather on the comparison strategy used by the participant.

Whereas the previous studies used the presence of a numerical distance effect on response times to serve as a processing signature, Meert et al. (2009) took a fundamentally different approach. In their study, Meert et al. used implicit priming as a marker for fraction processing type. They presented participants with two interleaved tasks; every fraction comparison trial was followed immediately by a whole number comparison trial, where participants were asked to choose the larger of two whole numbers (e.g., 4 versus 8). Meert et al. found that when the presented whole numbers matched the fraction components from the previous trial, there was a significant priming effect. Specifically, when the fraction pair had the same denominator and the subsequent whole number trial used the same numerators as in the preceding fraction trial, the responses were faster compared to control trials. For example, consider the fraction pair $3 / 8$ versus $5 / 8$, from which a participant would choose $5 / 8$ as the larger fraction. If the whole number pair 3 versus 5 was presented on the next trial (i.e., matching the numerators from the preceding fraction pair), participants tended to respond faster compared to conditions where the whole numbers did not match any of the earlier fraction components. Meert et al. (2009) conjectured that this facilitative priming effect was due to residual activation left over from directly comparing the numerators in the fraction pair.

On the other hand, when the fraction pair had the same numerator and the subsequent whole number trial used the same denominators as in the preceding fraction trial, the responses were slower compared to control trials. For example, consider the fraction pair $3 / 8$ versus $3 / 5$, from which a participant would choose $3 / 5$ as the larger fraction. If the whole number pair 8 versus 5 was presented on the next trial - matching the denominators from the preceding fraction pair - participants tended to respond more slowly compared to conditions where the whole numbers did not match any of the earlier fraction components. For these trials, Meert et al. (2009) explained that this slowdown happened because participants had used a "smaller denominator = larger fraction" strategy on the earlier fraction pair. This strategy resulted in a residual activation of a representation " $5>8$ ", which needed to be inhibited in order to then correctly activate the correct representation " $5<8$ " needed for the whole number comparison.

This pattern of priming effects led Meert et al. (2009) to conclude that in all cases participants were forming representations of the fraction components, and these activated representations either facilitated or interfered (depending on fraction type) with the subsequent whole number comparison.

Since the original study of Meert et al. (2009), this implicit priming paradigm has seen little use in the mathematical cognition literature. The purpose of the present study was to replicate the work of Meert et al. (2009), with one methodological addition. If participants are engaging in decomposed processing (i.e., forming separate representations of fraction components), we expect to see similar priming signatures on the whole number comparison task. On the other hand, if participants are using purely holistic strategies, we should see no such priming effects on the subsequent whole number comparisons. 
The methodological addition concerns how we index support for null effects. Meert et al. (2009) depended on the presence of a null effect to justify using one of their experimental conditions as a baseline for measuring priming effects. However, traditional hypothesis testing with $p$-values cannot be used as evidence for null effects (Masson, 2011; Wagenmakers, 2007). The reason for this is that absence of evidence for an effect is not equivalent to evidence for the absence of an effect (Ly, Etz, Marsman, \& Wagenmakers, 2019; Wagenmakers, Verhagen, \& Ly, 2016). Whereas a large $p$-value implies that an experimenter cannot reject the null (i.e., absence of evidence), this does not justify affirming that the null is true (i.e., evidence of absence). To circumvent this problem, we employed Bayesian hypothesis testing (Wagenmakers et al., 2017) in our analysis. Instead of indirectly gaining evidence for effects by rejecting a null hypothesis, the Bayesian approach allows us to directly compute posterior probabilities (i.e., probabilities of hypotheses after observing data) of the null and/or alternative hypothesis for each test.

\section{Method}

\section{Participants}

Thirty-two undergraduate psychology students (29 female, mean age $=24.9$ years, age range 18 to 58) participated in this experiment in exchange for partial course credit in their psychology courses. The experiment was reviewed and determined exempt by the Institutional Review Board at Tarleton State University.

\section{Materials and procedure}

Participants were each presented with 256 numerical comparison trials. On each trial, participants were asked to choose the larger of two fractions or two whole numbers. The trials were paired in the sense that a fraction trial always preceded a corresponding whole number trial. Thus, there were 128 fraction comparison trials and 128 whole number comparison trials.

Fraction pairs were taken from the stimulus list of Meert et al. (2009). They consisted of 32 pairs of same numerator fractions and 32 pairs of same denominator fractions, each presented twice in counterbalanced order. To keep the fractions as similar as possible across fraction type, the components used as denominators in a pair of same numerator fractions would also be used as numerators in a pair of same denominator fractions. All of the fractions used were irreducible (i.e., had no common factors) and excluded the use of 10 as a denominator. All fractions were proper (i.e., numerical magnitude less than one), and the natural number components ranged from 2 to 19. The use of irreducible fractions in the experiment allowed control of variability due to the possibility of participants simplifying the fractions, which could possibly mask any numerical distance or priming effects. Each fraction pair group had the larger fraction presented on the left in half of the pairs and on the right in the other half. 
Whole number pairs were constructed from the fraction pairs based on four different priming conditions, each of which resulted from crossing the variables of fraction type (same denominator, same numerator) and priming type (specific, nonspecific). Same denominator fractions had the form a/x_b/x; for example, 5/9 vs. 7/9. Same numerator fractions had the form $\mathrm{x} / \mathrm{a} \_\mathrm{x} / \mathrm{b}$; for example, $2 / 7$ vs. $2 / 3$. For specific priming trials, the corresponding whole number task would use the a and b components of the preceding fraction trial (e.g., the numerators that were presented in the same denominator pairs or the denominators that were presented in the same numerator pairs). The whole numbers were presented in the same spatial arrangement as they were in the preceding fraction trial. For nonspecific priming trials, the whole number task would use different numbers from any that were presented in the preceding fraction comparison trial.

The experimental trials were presented on a 20" iMac using SuperLab 5 software. Participants were seated in front of the computer with a viewing distance of approximately $60 \mathrm{~cm}$. Responses were indicated on a Cedrus RB-470 response pad, with the left-most button used to indicate that the left number was largest, whereas the right-most button was used to indicate that the right number was largest. The Cedrus response pad allowed us to record response times to approximately $2 \mathrm{~ms}$ accuracy. Stimuli were presented in white text on a black background. Each trial consisted of six components in the following order: (1) a fixation cross (+) presented in the center of the screen for $300 \mathrm{~ms}$; (2) a blank screen presented for $500 \mathrm{~ms}$; (3) a fraction pair; (4) a blank screen presented for $500 \mathrm{~ms}$; (5) an integer pair; and (6) a blank screen presented for $1500 \mathrm{~ms}$. For the numerical stimuli, participants were asked to indicate as quickly as possible the larger number from the pair presented on the screen. All number pairs were presented in the center of the screen and spanned a width of $13 \mathrm{~cm}$ (a horizontal visual angle of $12.4^{\circ}$ ). Fraction pairs spanned a height of $7 \mathrm{~cm}$ (a vertical visual angle of $7^{\circ}$ ), whereas integer pairs spanned a height of $2 \mathrm{~cm}$ (a vertical visual angle of $2^{\circ}$ ). Each experimental session was scheduled for 30 minutes; most participants needed only 15-20 minutes to complete all trials.

\section{Results}

\section{Data preparation}

Participants completed a total of 8,192 experimental trials. Four participants had error rates near $50 \%$ on the fraction task, so we excluded their data from further analysis. For the whole number task, the remaining 28 participants each contributed 128 trials, giving us a total of 3,584 trials for analysis. Thirty errors ( $0.8 \%$ of trials) and 4 trials that exceeded $5,000 \mathrm{~ms}$ were excluded, leaving 3,554 trials for analysis. The response times from these trials were collapsed (via the median) into 112 design cells, resulting from crossing the factors of participant $(N=28)$, fraction type (same denominator, same numerator), and priming type (specific, nonspecific). 


\section{Bayesian hypothesis testing}

Since Meert et al. used $t$-tests in their original paper, we used the default Bayesian $t$-test (Faulkenberry, 2019a; Rouder, Speckman, Sun, Morey, \& Iverson, 2009). The default Bayesian t-test works by first defining two competing hypotheses about effect size. Under the alternative hypothesis $\mathcal{H}_{1}$, the effect size $\delta$ is assumed to be distributed as a Cauchy distribution $^{1}$ with scale $1 / \sqrt{2}=0.707$ (Rouder et al., 2009). That is, we write

$$
\mathcal{H}_{1}: \delta \sim \text { Cauchy }\left(\frac{1}{\sqrt{2}}\right) \text {. }
$$

The Cauchy distribution is equivalent to a $t$-distribution with one degree of freedom. As such, the shape is similar to a normal distribution - i.e., peaked at 0 and symmetric - but the Cauchy distribution's tails are much fatter than those of the normal distribution. Our choice of prior and scale allows us to mathematically express our prior belief about the effect sizes we expect to observe in our experiment. Here, we would expect the most likely observed effect size to be 0 , and $50 \%$ of the observed effect sizes should range between -0.707 and +0.707 . Once we specify the prior distribution for effect sizes under $\mathcal{H}_{1}$, it is easy to define $\mathcal{H}_{0}$ by setting the effect size equal to 0 :

$$
\mathcal{H}_{0}: \delta=0
$$

After making these prior specifications of $\mathcal{H}_{1}$ and $\mathcal{H}_{0}$, we are ready to confront these hypotheses with data and find out which one best predicts our observed data. The predictive adequacy of each hypothesis is tested using Bayes factors (Kass \& Raftery, 1995). A Bayes factor - denoted $\mathrm{BF}_{10}$ - is defined as the relative likelihood of the observed data under the two hypotheses $\mathcal{H}_{1}$ and $\mathcal{H}_{0}$. For example, a Bayes factor of $\mathrm{BF}_{10}=8$ means that the observed data are 8 times more likely under the alternative hypothesis $\mathcal{H}_{1}$ than the null hypothesis $\mathcal{H}_{0}$. Similarly, $\mathrm{BF}_{01}=8$ would mean that the observed data are 8 times more likely under the null hypothesis $\mathcal{H}_{0}$ than the alternative hypothesis $\mathcal{H}_{1}$. Note that the Bayes factor is calculated as the ratio of marginal likelihoods of the observed data under each of these hypotheses. This means that the Bayesian $t$-test simultaneously assesses the adequacy of both $\mathcal{H}_{0}$ and $\mathcal{H}_{1}$ as predictive models of our observed data, something that a frequentist $t$-test does not do.

Bayes factors also quantify the factor by which the prior odds in favor of either $\mathcal{H}_{0}$ or $\mathcal{H}_{1}$ are changed after observing data, so it is straightforward to convert the Bayes factor to a posterior probability for either $\mathcal{H}_{0}$ or $\mathcal{H}_{1}$ (assuming $\mathcal{H}_{0}$ and $\mathcal{H}_{1}$ have equal prior odds; see Masson, 2011; Faulkenberry, 2019b). Specifically, if the Bayes factor tells us that the data are more likely under $\mathcal{H}_{0}$, we can compute

$$
p\left(\mathcal{H}_{0} \mid \text { data }\right)=\frac{\mathrm{BF}_{01}}{1+\mathrm{BF}_{01}} .
$$

\footnotetext{
${ }^{1}$ The scale value $0.707=1 / \sqrt{2}$ is recommended as a default because the resulting Bayes factor has a number of desirable properties; see Rouder et al., 2009, for details.
} 


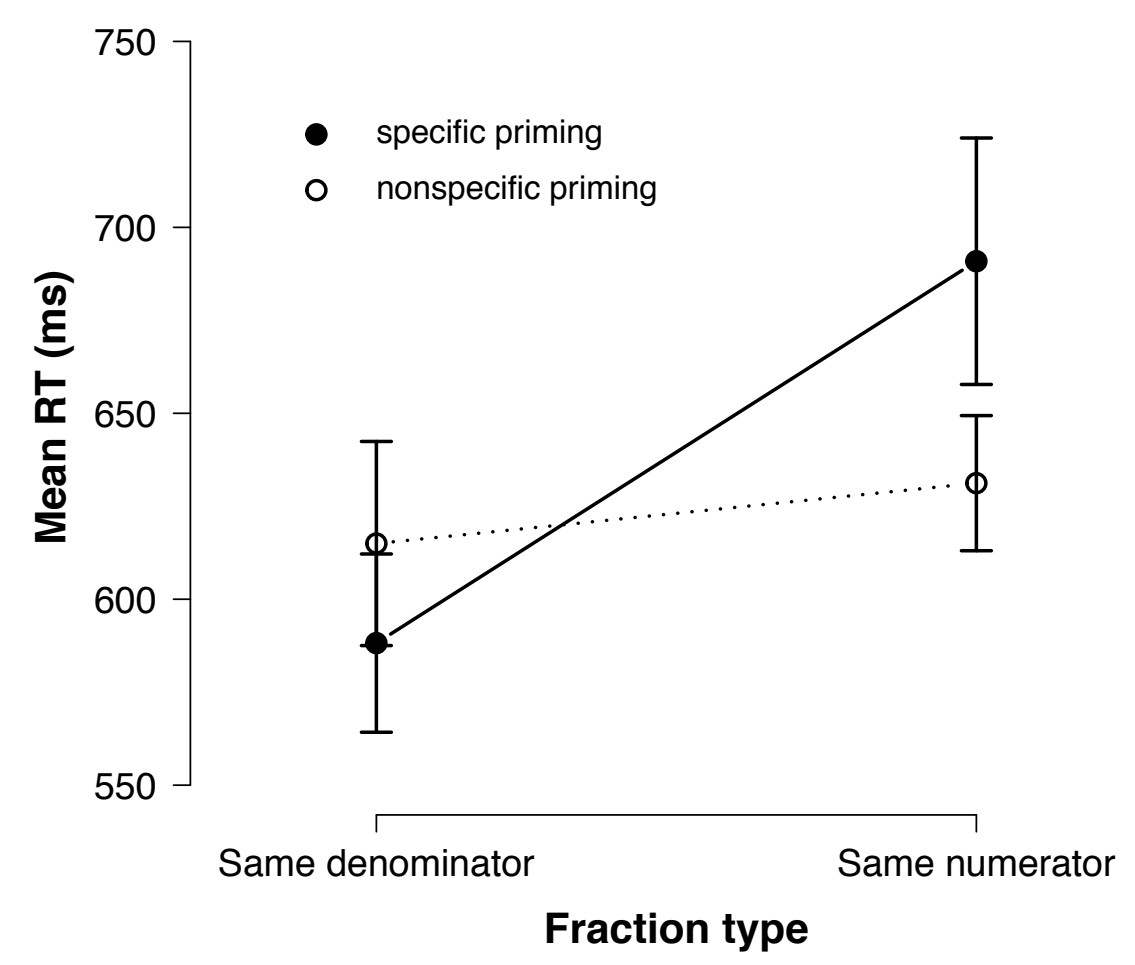

Figure 1. Mean response times on the whole number comparison task as a function of fraction type (same denominator, same numerator) and priming type (specific, nonspecific). Error bars represent within-subject $95 \%$ confidence intervals as recommended by Morey (2008).

On the other hand, if we find that the data are more likely under $\mathcal{H}_{1}$, we can compute

$$
p\left(\mathcal{H}_{1} \mid \text { data }\right)=\frac{\mathrm{BF}_{10}}{1+\mathrm{BF}_{10}} .
$$

All Bayes factors were computed using the free software package JASP (JASP Team, 2019; Marsman \& Wagenmakers, 2016), freely downloadable from www.jasp-stats.org.

\section{Response time analysis}

Mean response times in the four experimental conditions can be seen in Figure 1, which follows the same general pattern found in Meert et al. (2009). Our first aim was to conduct a manipulation check and demonstrate that priming did occur in our experimental design; that is, when participants first compared two fractions, there was a priming effect in the subsequent whole number comparison trials in the specific priming condition, and critically, that there was a null priming effect in the nonspecific condition.

We found that in the specific priming condition, whole number comparisons that followed same denominator trials $(M=588 \mathrm{~ms}, S E=25 \mathrm{~ms})$ were faster than trials 
which following same numerator trials $(M=691 \mathrm{~ms}, S E=39 \mathrm{~ms})$. These data were overwhelmingly supportive of the alternative hypothesis predicting faster responses for same denominator trials than for same numerator trials, $\mathrm{BF}_{10}=503.0$. This Bayes factor indicates that the observed data are 503 times more likely under the alternative hypothesis than the null hypothesis. Assuming equal prior odds for $\mathcal{H}_{0}$ and $\mathcal{H}_{1}$, this is equivalent to a posterior probability of 0.998 in favor of the alternative hypothesis $\mathcal{H}_{1}$. However, for the nonspecific priming condition, we found no difference in response times between same denominator trials $(M=615 \mathrm{~ms}, S E=30 \mathrm{~ms})$ and same numerator trials $(M=631 \mathrm{~ms}, S E=33 \mathrm{~ms})$. The Bayes factor in favor of this null effect was $\mathrm{BF}_{01}=3.35$, indicating that the observed data were 3.35 times more likely under the null hypothesis than the alternative hypothesis. Assuming equal prior odds for $\mathcal{H}_{0}$ and $\mathcal{H}_{1}$, this converts to a posterior probability of 0.770 in favor of the null hypothesis $\mathcal{H}_{0}$. Thus, since whole number performance does not differ as a function of fraction type in nonspecific priming trials, it makes sense to use the nonspecific priming condition as a baseline for the specific priming effects.

Now we consider the effects of priming as a function of fraction type. To this end, we consider differences in response time between trials in the specific priming condition and trials in the baseline, nonspecific priming condition. For whole number comparisons primed by same denominator fraction comparisons, there was a speed-up compared to baseline ( $M=615 \mathrm{~ms}$ for baseline versus $M=588 \mathrm{~ms}$ for primed trials). The Bayes factor for the alternative hypothesis predicting such a speedup was $\mathrm{BF}_{10}=2.84$; assuming equal prior odds for $\mathcal{H}_{0}$ and $\mathcal{H}_{1}$, this Bayes factor converts to a posterior probability of 0.740 in favor of the alternative hypothesis $\mathcal{H}_{1}$. On the other hand, whole number comparisons following same numerator trials showed the opposite pattern. When primed by same numerator fraction comparisons, participants' responses were slowed compared to baseline $(M=691$ ms for primed trials compared to $M=631 \mathrm{~ms}$ for baseline). The Bayes factor in favor of the alternative hypothesis predicting this slowdown was $\mathrm{BF}_{10}=298.1$, which converts to a posterior probability of 0.997 in favor of the alternative hypothesis $\mathcal{H}_{1}$.

In summary, we found positive evidence for the priming effects described by Meert et al. (2009). Same denominator fraction comparisons tended to speed up subsequent whole number comparisons, whereas same numerator fraction comparisons tended to slow down the subsequent whole number comparisons. This would only be possible if participants were forming representations of the individual fraction components, and these activations were carried downstream into the whole number comparison. Thus, our results lend further support to the idea that fraction comparisons involve decomposed processing.

\section{Discussion}

The purpose of the present study was to investigate the mental representations formed when people think about fractions. Specifically, we aimed to test whether participants formed decomposed representations of fractions (i.e., whether they formed representations of numerators and/or denominators) when engaged in a fraction comparison task. To do this, we used the implicit priming paradigm first applied by Meert et al. (2009), who found that when whole number comparisons immediately followed fraction comparisons with the same two numbers in the numerators, there was a speed-up over baseline in the whole 
number task. However, when the fraction comparison involved the same two numbers in the denominators, there was a slowdown in the whole number task. From this, Meert et al. (2009) concluded that participants were forming separate representations of the components in the fractions that either facilitated or interfered with the subsequent whole number task. That is, participants exhibited decomposed processing with fractions.

In our replication of Meert et al. (2009), we found roughly the same results. First, we demonstrated that the implicit priming paradigm does indeed work in the context of mathematical cognition. That is, in the specific priming condition, where the to-be-compared components of the fraction pair exactly matched the two numbers that were compared in the subsequent whole number comparison, there was a marked difference in response times between fraction types. However, in the nonspecific priming conditions, where there was no such match between the fraction components and the subsequent whole number comparison, there was no difference in response times between fraction types. We used Bayesian inference to confirm this null effect, which extends what Meert et al. (2009) were able to conclude from their original, purely frequentist analyses.

After confirming that response times for nonspecific priming trials did not differ between fraction types, we were able to confirm the predictions of Meert et al. (2009) regarding the specific direction of the priming effects. Specifically, in same denominator trials, there was a relative speed-up in the subsequent whole number comparison. This reflects a facilitation effect; if participants are forming decomposed representations of the numerators in the fraction pair, the residual activation of these numerators then facilitates the comparison of the whole numbers in the very next trial. On the other hand, in same numerator trials, the opposite pattern occurs. In these fraction pairs, the nature of the task is different. For example, consider the pair $2 / 7$ versus $2 / 5$. If participants are forming decomposed representations of the 7 and the 5 in the denominators, the context of fraction comparison dictates that participants should adopt a "smaller is bigger" strategy and conclude that the fraction with the smaller denominator $(2 / 5)$ is the larger fraction (Faulkenberry \& Pierce, 2011). This results in an inhibitory effect on the subsequent whole number comparison. In whole number comparison, the comparison strategy will be opposite to what was employed for the fractions. Thus, any residual activation from the representation of the denominators 5 and 7 will have to decay before the participant can correctly judge that 7 is greater than 5 in a whole number context. The result is that we observe increased response times in the whole number comparison task (relative to baseline).

In all, these data lend support to models of fraction representation which hypothesize decomposed processing of fractions (e.g., Bonato et al., 2007; Faulkenberry et al., 2015; Faulkenberry \& Pierce, 2011). More broadly, this research fits in with similar studies of holistic versus decomposed processing in other numerical contexts, including two-digit number comparison (e.g., Verguts \& De Moor, 2005; Faulkenberry, Cruise, \& Shaki, 2020; Nuerk, Weger, \& Willmes, 2001). Further, we demonstrated the pragmatic advantages of Bayesian inference as a tool for assessing null effects in psychological research. In future work, we hope to further elucidate the nature of fraction representations in more broadly defined contexts, such as using more complex fractions (e.g., 13/24) or embedding fractions in word problems (e.g., one part out of five). In addition, it will be interesting to investigate 
the implicit priming paradigm as a tool for testing theories in mathematical cognition, potentially validating its use as a measurement tool for indexing individual differences in mathematical skill. 


\section{References}

Bonato, M., Fabbri, S., Umiltà, C., \& Zorzi, M. (2007). The mental representation of numerical fractions: Real or integer? Journal of Experimental Psychology: Human Perception and Performance, 33(6), 1410-1419. https://doi.org/10.1037/0096-1523. 33.6.1410

Faulkenberry, T. J. (2019a). A tutorial on generalizing the default Bayesian $t$-test via posterior sampling and encompassing priors. Communications for Statistical Applications and Methods, 26(2), 217-238. https://doi.org/10.29220/csam.2019.26.2.217

Faulkenberry, T. J. (2019b). Estimating evidential value from analysis of variance summaries: A comment on Ly et al. (2018). Advances in Methods and Practices in Psychological Science, 2(4), 406-409. https://doi.org/10.1177/2515245919872960

Faulkenberry, T. J., Cruise, A., \& Shaki, S. (2020). Task instructions modulate unit-decade binding in two-digit number representation. Psychological Research, 84(2), 424-439. https://doi.org/10.1007/s00426-018-1057-9

Faulkenberry, T. J., Montgomery, S. A., \& Tennes, S.-A. N. (2015). Response trajectories reveal the temporal dynamics of fraction representations. Acta Psychologica, 159, 100-107. https://doi.org/10.1016/j.actpsy.2015.05.013

Faulkenberry, T. J., \& Pierce, B. H. (2011). Mental representations in fraction comparison: Holistic versus component-based strategies. Experimental Psychology, 58 (6), 480-489. https://doi.org/10.1027/1618-3169/a000116

Gabriel, F. C., Coché, F., Szücs, D., Carette, V., Rey, B., \& Content, A. (2013). A componential view of children's difficulties in learning fractions. Frontiers in Psychology, 4, 715. https://doi.org/10.3389/fpsyg.2013.00385

Ganor-Stern, D., Karasik-Rivkin, I., \& Tzelgov, J. (2011). Holistic representation of unit fractions. Experimental Psychology, 58(3), 201-206. https://doi.org/10.1027/ 1618-3169/a000086

Hoffer, T. B., Venkataraman, L., Hedberg, E. C., \& Shagle, S. (2007). Final report on the national survey of algebra teachers for the national math panel. National Opinion Research Center at the University of Chicago.

Huber, S., Moeller, K., \& Nuerk, H.-C. (2014). Adaptive processing of fractions - Evidence from eye-tracking. Acta Psychologica, 148, 37-48. https://doi.org/10.1016/j.actpsy. 2013.12.010

JASP Team. (2019). JASP (Version 0.11.1.0)[Computer software]. Retrieved from https: //jasp-stats.org/

Kass, R. E., \& Raftery, A. E. (1995). Bayes factors. Journal of the American Statistical Association, 90(430), 773-795. https://doi.org/10.1080/01621459.1995.10476572

Kutner, M., Greenberg, E., Jin, Y., Boyle, B., Hsu, Y., \& Dunleavy, E. (2007). Literacy in everyday life: Results from the 2003 National Assessment of Adult Literacy. NCES 
2007-480. Retrieved from http://nces.ed.gov/Pubs2007/2007480.pdf.

Ly, A., Etz, A., Marsman, M., \& Wagenmakers, E.-J. (2019). Replication Bayes factors from evidence updating. Behavior Research Methods, 51(6), 2498-2508. https: //doi.org/10.3758/s13428-018-1092-x

Marsman, M., \& Wagenmakers, E.-J. (2016). Bayesian benefits with JASP. European Journal of Developmental Psychology, 14(5), 545-555. https://doi.org/10.1080/17405629. 2016.1259614

Masson, M. E. J. (2011). A tutorial on a practical Bayesian alternative to null-hypothesis significance testing. Behavior Research Methods, 43(3), 679-690. https://doi.org/10. 3758/s13428-010-0049-5

Meert, G., Grégoire, J., \& Noël, M.-P. (2009). Rational numbers: Componential versus holistic representation of fractions in a magnitude comparison task. Quarterly Journal of Experimental Psychology, 62(8), 1598-1616. https://doi.org/10.1080/ 17470210802511162

Meert, G., Grégoire, J., \& Noël, M.-P. (2010). Comparing 5/7 and 2/9: Adults can do it by accessing the magnitude of the whole fractions. Acta Psychologica, 135(3), 284-292. https://doi.org/10.1016/j.actpsy.2010.07.014

Morey, R. D. (2008). Confidence intervals from normalized data: A correction to Cousineau (2005). Tutorials in Quantitative Methods for Psychology, 4(2), 61-64. https: //doi.org/10.20982/tqmp.04.2.p061

Nuerk, H.-C., Weger, U., \& Willmes, K. (2001). Decade breaks in the mental number line? Putting the tens and units back in different bins. Cognition, 82(1), B25-B33. https://doi.org/10.1016/s0010-0277(01)00142-1

Obersteiner, A., Dooren, W. V., Hoof, J. V., \& Verschaffel, L. (2013). The natural number bias and magnitude representation in fraction comparison by expert mathematicians. Learning and Instruction, 28, 64-72. https://doi.org/10.1016/j.learninstruc.2013.05. 003

Obersteiner, A., \& Tumpek, C. (2015). Measuring fraction comparison strategies with eye-tracking. ZDM Mathematics Education, 48(3), 255-266. https://doi.org/10. 1007/s11858-015-0742-z

Rouder, J. N., Speckman, P. L., Sun, D., Morey, R. D., \& Iverson, G. (2009). Bayesian $t$-tests for accepting and rejecting the null hypothesis. Psychonomic Bulletin 8 Review, 16 (2), 225-237. https://doi.org/10.3758/pbr.16.2.225

Schneider, M., \& Siegler, R. S. (2010). Representations of the magnitudes of fractions. Journal of Experimental Psychology: Human Perception and Performance, 36(5), 1227-1238. https://doi.org/10.1037/a0018170

Verguts, T., \& De Moor, W. (2005). Two-digit comparison: Decomposed, holistic, or hybrid? Experimental Psychology, 52(3), 195-200. https://doi.org/10.1027/1618-3169.52.3. 195 
Wagenmakers, E.-J. (2007). A practical solution to the pervasive problems of $p$ values. Psychonomic Bulletin \& Review, 14(5), 779-804. https://doi.org/10.3758/bf03194105

Wagenmakers, E.-J., Marsman, M., Jamil, T., Ly, A., Verhagen, J., Love, J., ... Morey, R. D. (2017). Bayesian inference for psychology. Part i: Theoretical advantages and practical ramifications. Psychonomic Bulletin $\&$ Review, 25(1), 35-57. https: //doi.org/10.3758/s13423-017-1343-3

Wagenmakers, E.-J., Verhagen, J., \& Ly, A. (2016). How to quantify the evidence for the absence of a correlation. Behavior Research Methods, 48(2), 413-426. https: //doi.org/10.3758/s13428-015-0593-0

Zhang, L., Fang, Q., Gabriel, F. C., \& Szücs, D. (2014). The componential processing of fractions in adults and children: Effects of stimuli variability and contextual interference. Frontiers in Psychology, 5. https://doi.org/10.3389/fpsyg.2014.00981

Zhang, L., Xin, Z., Li, F., Wang, Q., Ding, C., \& Li, H. (2012). An ERP study on the processing of common fractions. Experimental Brain Research, 217(1), 25-34. https://doi.org/10.1007/s00221-011-2969-4 